\title{
Afectaciones de la pandemia del covid-19 en los corredores comerciales y turísticos de ciudad Juárez
}

\author{
Aida-Yarira Reyes Escalante \\ aida.reyes@uacj.mx \\ Universidad Autónoma de Ciudad Juárez, México \\ Andrea Paulina Rodríguez Hernández \\ A1147234@alumnos.uacj.mx \\ Universidad Autónoma de Ciudad Juárez, México \\ Carlos Jesús González Macías* \\ cgonzalez@uacj.mx \\ Universidad Autónoma de Ciudad Juárez, México \\ *autor de correspondencia \\ Diego Adiel Sandoval Chávez \\ dsandoval@itcj.edu.mx \\ Tecnológico Nacional de México, campus ITCJ, México
}

\section{RESUMEN}

Los corredores comerciales y turísticos son por naturaleza generadores de recursos económicos, sociales y ambientales, como una alternativa comercial que conjunta elementos claves para el desarrollo de un sector y de una comunidad, representando atractivo turístico de dinámicas únicas. En el marco de la pandemia COVID -19, los corredores comerciales reflejan la crisis que se viven; en Ciudad Juárez se han visto afectados, sin embargo, continúan observando actividad. El objetivo fue conocer las estrategias implementadas para continuar la actividad económica de dichos corredores. El estudio es descriptivo, transversal y de metodología mixta, para aproximar y conocer las estrategias implementadas en siete corredores comerciales, por medio de entrevista a profundidad y cuestionarios. Los resultados reflejaron las condiciones posteriores a los cierres totales establecidos por los tres niveles de gobierno, revelando que dichos corredores se vieron seriamente afectados; y en todos ellos se aplicaron estrategias generales de protección a los contagios.

Palabras clave: corredores comerciales; afectaciones económicas y sociales; covid-19; ciudad Juárez. 


\title{
Affectations from covid-19 pandemic to commercial and touristic corridors from ciudad Juarez
}

\begin{abstract}
Commercial and touristic corridors are, by their nature, generators of economic, social and environmental resources, as a commercial alternative that conjuncts key elements for sector and community developments, representing a unique dynamics touristic attraction. In the frame of COVID-19 pandemic, commercial corridors reflect the experienced crisis; in Ciudad Juarez the have been affected, but nevertheless they continue in activities. The object was to acknowledge the enforced strategies to continue activities in those corridors. Is a descriptive, transversal and mixed-method study, to approach and acknowledge the enforced strategies in seven commercial corridors, through in-depth interview and questionnaires. Findings showed the posterior conditions after total shutdowns stablished by the three government levels, revealing that those corridors were seriously affected; and in all of them were enforced general strategies for contagion protection.
\end{abstract}

Keywords: commercial corridors; economic and social affectations; covid-19; ciudad Juarez.

Artículo recibido: 02 noviembre. 2021 Aceptado para publicación: 28 noviembre 2021 Correspondencia: cgonzalez@uacj.mx Conflictos de Interés: Ninguna que declarar 


\section{INTRODUCCIÓN}

La globalización de la economía es un proceso en donde los límites fueron superados, ya que los accesos basados en el desarrollo tecnológico y la existencia de productos, aplicaciones y dispositivos permitieron que las actividades económicas dejaran de ser locales o regionales, y se encaminaran hacia lo global. Las economías mundiales tienen relaciones entre todas: lo que sucede a unas, repercute en otras.

La enfermedad causada por el COVID-19, o novel coronavirus, derivó en una pandemia global de enfermedad respiratoria aguda, que filogenéticamente está estrechamente relacionado con el SARS-CoV. Comenzó en diciembre de 2019 en Wuhan, provincia de Hubei en China, y fue declarada pandemia global hacia el 11 de marzo de 2020. La mayoría de los casos de COVID-19 tienen incidencia en personas de edad adulta (Castro, 2020). A partir de la declaración del brote de la enfermedad por la Organización Mundial de la Salud (OMS, 2021), los países cerraron sus fronteras con la finalidad de evitar contagios, y con ello los negocios y las empresas iniciaron un proceso de supervivencia dentro de las condiciones más extremas.

El virus fue identificado como parte de la familia Coronavirus, reconocido por su rapidez de contagio y afectaciones al sistema respiratorio, con síntomas de fiebre y dolor muscular, pérdida del sentido gustativo y considerada una enfermedad leve o moderada del tracto respiratorio superior, similar a un resfriado común; sus efectos provocan la muerte dolorosa del paciente y los sobrevivientes quedan con secuelas secundarias. Las afectaciones por contagio a nivel mundial han causado pérdidas humanas millonarias y se han reflejado en los efectos económicos a todos los niveles. De acuerdo a la OMS (2021) en junio de 2021 se registraron los números más altos de nuevos casos: en India (1,364, 668 nuevos casos, 26\% de disminución), Brasil (420,981 nuevos casos, 7\% de disminución), Argentina (219,910 nuevos, casos; 3\% de aumento), Estados Unidos de Norteamérica (153,587 nuevos casos, $18 \%$ de disminución) y Colombia (150,517 nuevos casos, $40 \%$ de aumento)

El impacto de la crisis económica se sintió en la caída al Producto Interno Bruto (PIB) en los países de todo el mundo. El impacto negativo que el COVID-19 causó de forma global se tangibilizaron en las pérdidas financieras de la mayoría de las empresas, cierres masivos de negocios, el aumento de desempleados, el aumento de inseguridad por falta de solvencia económica de los hogares, falta de recursos e infraestructura de las ciudades, 
la deficiencia en el sistema de salud y el suministro de medicamentos, cierre masivo de organizaciones de apoyo social, entre otros. Dentro de los indicadores relacionados a las empresas, se identifican los daños a la actividad industrial, donde se dejó de producir, los suministros empezaron a faltar y muchos de ellos dejaron de ser producidos. En este escenario pesimista, se dio un aumento al desempleo y subempleo, agudizando las crisis económicas de los ciudadanos y su poder adquisitivo, y con ello el aumento a la crisis económica en todo el comercio (OMC, 2021).

Durante los periodos de más contagio en todos los países, se desarrollaron estrategias para evitar la propagación rápida del COVID-19; las dinámicas cotidianas fueron las más afectadas por el brote del virus, las personas en sus comportamientos y rutinas diarias se vieron alteradas por toques de queda, prohibición de visitas a lugares públicos y aglomerados, en muchos lugares se prohibió salir de sus casas o departamentos en su totalidad. Un tercio de la población mundial se encontró en situación de encierro total o parcial, y debido a esto las empresas consideradas no elementales o de necesidades primarias cerraron sus puertas, retirando sus servicios o modificándolos, con la finalidad de seguir en el mercado. La sociedad perdió su libertad, su salud, sus empleos, sus relaciones sociales, sus hogares. Uno de los aumentos identificados durante el encierro, fue el desarrollo de la educación virtual, consumo de bienes y servicios en línea, y el aumento del autoempleo o subempleo conocido como trabajo desde el hogar (home office) (SER, 2021).

Los empresarios de todo el mundo se enfrentaron a los efectos de la crisis económica, viéndose obligados a generar cambios para proteger sus empresas, tal como lo mencionan Kunkertz et al. (2020), al indicar que el bloqueo amenaza la existencia de muchas startups innovadoras, y sugieren que, aunque las empresas emergentes están aprovechando con éxito los recursos de que disponen como primera respuesta a la crisis, su crecimiento y su potencial de innovación están en peligro, aseverando la necesidad de políticas de ayuda a las startups para garantizar una rápida recuperación y crecimiento.

Collison (2020) indica que muchos consumidores de productos minoristas fueron beneficiados al vender sus productos a bajos precios, observándose un aumento en el consumo desde el hogar, pequeños comercios y entrega de comida casera. Las aerolíneas cayeron un $19.1 \%$ del 19 de febrero a marzo del 2020 debido a la prohibición de viajes. Otra de las industrias que más ha sufrido ha sido la de los eventos: conferencias, festivales 
de música, maratones y los eventos deportivos más grandes del mundo fueron cancelados o pospuestos para más adelante. Las acciones de hoteles, resorts y cruceros cayeron un 22.2\% desde el 19 de febrero marzo 2020. Las búsquedas de servicios de entrega de comida se incrementaron un $80 \%$ en restaurantes y centros comerciales durante el 2020 (ver Figura 1).

Figura 1. Crecimiento de servicios de entrega de comidas

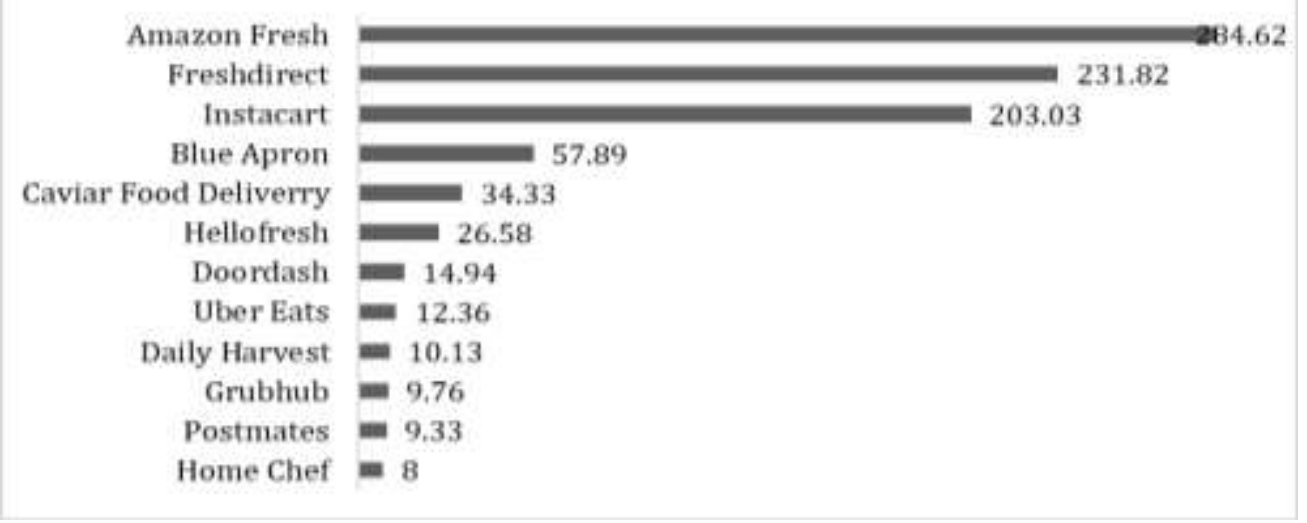

Fuente: Collison (2020).

México, como el resto del mundo, se encuentra en una severa crisis económica agravada por la pandemia COVID-19, y sus manifestaciones se observan en el desplome de precios del petróleo, la caída en la actividad económica en 2019 y 2020, el alza en la cotización del dólar, cierre de empresas y pérdida de empleos, perdida de consumo y aumento de precios de casi todos los alimentos y servicios (INEGI, 2021). Las estadísticas de los contagios (DGE, 2021; INEGI, 2021) revelan que entre los meses de enero y febrero del 2021 fueron de mayor contagio (ver Figura 2 y Figura 3), y las estadísticas generales (INEGI, 2021) reflejan 228 mil muertes (ver Tabla 1). La pérdida de empleos se encuentra reflejada en la Tabla 2 (INEGI, 2021).

Figura 2. Estadísticas de contagios mensuales en México

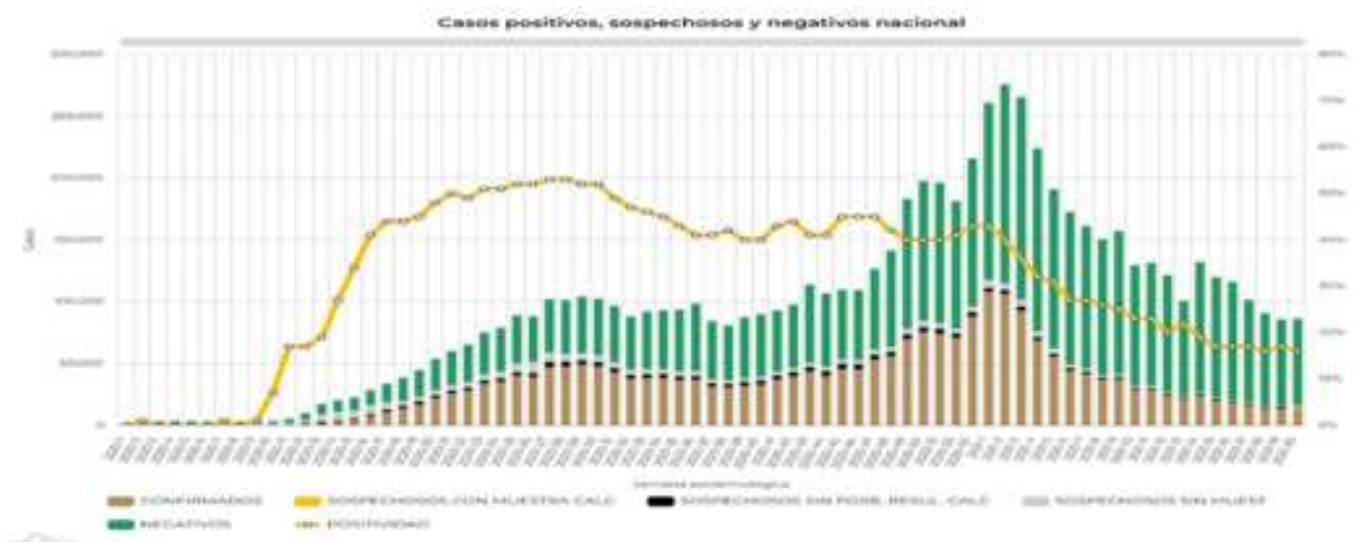

Fuente: Dirección General de Epidemiología (2021). 
Figura 3. Situación de los estados de México ante el Covid-19

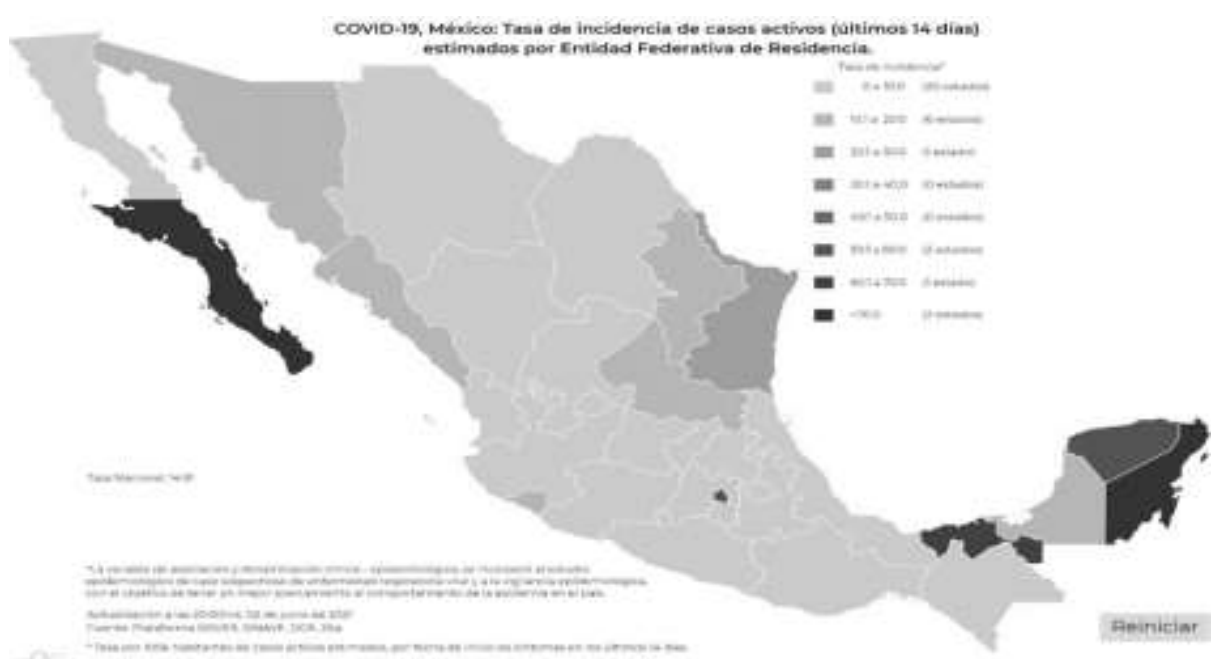

Fuente: INEGI (2021).

Tabla 1. Indicadores de Covid-19 en México

\begin{tabular}{|c|c|c|c|}
\hline $\begin{array}{c}\text { Casos } \\
2.42 \mathrm{M} \\
2,420,000 \\
+6,917 \\
+6,917 \\
\end{array}$ & \multicolumn{2}{|c|}{$\begin{array}{c}\text { Personas recuperadas } \\
1.93 \mathrm{M} \\
1,930,000 \\
+2,141 \\
+2,141 \\
\end{array}$} & $\begin{array}{c}\text { Muertes } \\
228 \mathrm{k} \\
228,000 \\
+4,272 \\
+4,272 \\
\end{array}$ \\
\hline Ubicación & Casos & Personas recuperadas & Muertes \\
\hline $\begin{array}{l}\text { Ciudad de } \\
\text { México }\end{array}$ & $\begin{array}{c}660 \mathrm{k} \\
660,000 \\
+1,708 \\
+1,708\end{array}$ & - & $\begin{array}{l}34,206 \\
34,206 \\
+1,003 \\
+1,003\end{array}$ \\
\hline $\begin{array}{l}\text { Estado de } \\
\text { México }\end{array}$ & $\begin{array}{c}252 \mathrm{k} \\
252,000 \\
+2,093 \\
+2,093\end{array}$ & - & $\begin{array}{r}36,907 \\
36,907 \\
+1,933 \\
+1,933\end{array}$ \\
\hline Guanajuato & $\begin{array}{c}132 \mathrm{k} \\
132,000 \\
+60 \\
+60\end{array}$ & - & $\begin{array}{c}10,914 \\
10,914 \\
+42 \\
+42\end{array}$ \\
\hline Nuevo León & $\begin{array}{c}124 \mathrm{k} \\
124,000 \\
+80 \\
+80\end{array}$ & - & $\begin{array}{c}9,562 \\
9,562 \\
+30 \\
+30\end{array}$ \\
\hline Jalisco & $\begin{array}{c}87,165 \\
87,165 \\
+85 \\
+85\end{array}$ & - & $\begin{array}{c}12,427 \\
12,427 \\
+50 \\
+50\end{array}$ \\
\hline
\end{tabular}

Nota: El signo "+" indica los casos nuevos que se reportaron 1 junio 2021. Fuente: Elaboración propia con base en INEGI (2021). 
Tabla 2. Situación del Mercado Laboral en México, 2019-2020 (millones de personas)

\begin{tabular}{|l|c|c|c|}
\hline \multicolumn{1}{|c|}{ Concepto } & Mayo 2019(ENOE) & Mayo 2020 (ETOE) & Cambio \\
\hline Población Ocupada & 55.0 & 43.6 & -11.4 \\
Subocupados & 4.3 & 13.0 & 8.7 \\
No Subocupados & 50.7 & 30.5 & -20.2 \\
\hline Población desocupada & 2.0 & 1.9 & -0.1 \\
\hline Población No & 37.7 & 50.4 & 12.7 \\
Económicamente Activa & 5.4 & 19.4 & 14.0 \\
$\quad$ Disponibles & 32.3 & 31.0 & -1.3 \\
$\quad$ No disponibles & $3.5 \%$ & $4.2 \%$ & 0.7 \\
\hline Tasa de desocupación & & & \\
\hline
\end{tabular}

Fuente: Elaboración propia con base en INEGI (2021).

\section{CORREDORES COMERCIALES}

Dentro de las zonas urbanas se generan espacios comerciales que se van consolidando debido a la oferta de bienes y servicios, como su estructura física y equipamiento; estas zonas han sido parte de las estrategias para detonar actividades económicas y son conocidas como corredores urbanos.

Bolaños y Reyes (2020) indican que el surgimiento de las zonas comerciales se va desarrollando al utilizar avenidas, calles o caminos transformándolos en espacios específicos para el comercio, industria, turismo, cultura, etc. Además, mencionan que los corredores urbanos son parte de las estrategias de ordenamiento y desarrollo urbano, buscando utilizar las actividades realizadas hacia el beneficio económico y social; se consideran relevantes para regenerar la zona, por lo que muchas ciudades buscan utilizarlos para obtener mayores y mejores beneficios.

Dichos corredores poseen una gran variedad de tipos, entre ellos: comerciales, de negocios, micro-industriales, de servicios públicos, turísticos y recreativos, deportivoculturales, turísticos y ecológicos. La existencia de estos corredores garantiza la derrama económica además de otros intereses, que son características intrínsecas al corredor.

Los corredores de tipo comercial y turístico deben tomar en cuenta diversas variables. Según las definiciones de la Organización de los Estados Americanos (OEA, 2021), el corredor comercial es un espacio homogéneo en el que por la cercana distancia de los atractivos y servicios se llega a una natural complementariedad. Por lo general, se agrega que rutas troncales efectivizan su integración y la jerarquía de los atractivos y productos determinan el rango de convocatoria de dicho espacio (Calaméo, 2021), además de tener vías de conexión entre las zonas, áreas, complejos, centros, conjuntos, atractivos 
turísticos, puertos de entrada del turismo receptivo y plazas emisoras del turismo interno, que funcionan como elementos estructuradores del espacio turístico. Adicionalmente, se debe considerar el uso actual que tienen, el ancho de la calle y la vegetación existente en el sector, ya que estas vialidades poseen el potencial para desarrollar actividades comerciales de baja, mediana y alta intensidad. A su vez, se debe satisfacer la demanda de los habitantes de las zonas colindantes, así como de las personas que frecuentan el sector con la finalidad de hacer eficiente el carácter del suelo en el lugar, proporcionando seguridad, funcionalidad, comodidad, oportunidad, y a la vez la diversidad de productos y servicios, para hacer del lugar un sector competitivo en esta materia

Los corredores comerciales permiten que los negocios minoristas detonen un plan estratégico para el desarrollo del sector donde se ubiquen. Este modelo consiste en organizar el sector con una estrategia común de ventas para lograr consolidar el comercio en un área, con la finalidad de brindar al cliente consumidor opciones cercanas, la seguridad de saber lo que allí va a encontrar lo que necesite, creando áreas con un atractivo particular que lo caracterice; con ellos se busca el desarrollo en calles dinámicas ya consolidadas y sectores con patrimonio histórico o cultural. Los corredores comerciales deben involucrar al conjunto de los propietarios del suelo con la visión de que las estrategias sean conjuntas en el beneficio de todos, que desarrollen un interés común por el impulso de la zona en conjunto.

La propuesta de un corredor comercial también debe incluir un modelo de desarrollo poseedor de propuestas donde se contemplen zonas habitacionales, en las cuales las casas sean parte fundamental de dicha propuesta; ello contribuirá a desarrollar corredores comerciales con más vida, manteniendo su estilo, imagen, identidad y sabor comunitario, donde todo el desarrollo comercial motive a los pequeños negocios locales a diseñar fachadas agradables para armonizar los paseos peatonales. La participación de la comunidad en corredores comerciales cumple con lo indicado por Ander-Egg (2000), al reconocer que la gente sabe, vive, siente, hace y quiere, reconociendo cotidianeidad motivando su participación; es decir, cosas para despertar un determinado interés, siendo este la atención a aquello que se percibe subjetivamente valioso y que tiene significación para la propia vida. Corredores comerciales y turísticos desde la vida diaria de sus habitantes, ventas de productos locales, actividades diarias y cultura local.

El interés por los corredores comerciales es evidente en las investigaciones realizadas en 
diversos países. Romero (2011) abordó un estudio relacionado con el corredor histórico en la Ciudad de México, planteando políticas públicas en la conformación del corredor urbano centro histórico de la ciudad de México-Santa Fe. Menciona que las ciudades crecen y se van creando en zonas antiguas que tienen características únicas, donde se pueden realizar intervenciones con megaproyectos financiados por capitales externos y, por consiguiente, se requiere de una la adecuación de instrumentos jurídicos, de planeación y de gestión urbana para que dichos proyectos tengan éxito. Moreno (2015) plantea el corredor industrial automotriz mediante la propuesta de un plan maestro para el Bajío. El estudio se basa en el Plan Nacional de Desarrollo (PND) (Secretaría de Gobernación, 2019), en el cual se reafirma el compromiso con el libre comercio, la movilidad de capitales y su regulación y promoción, logrando plantear la necesidad de involucrar a las autoridades y organizaciones industriales con la finalidad de lograr los cambios en las dinámicas territoriales para determinar la autorización de cambios de uso de suelo y los permisos de construcción. En el 2009, se desarrolló el proyecto relacionado con los corredores del TLCAN como rutas fundamentales para los flujos económicos mundiales. La vinculación de los corredores comerciales en Canadá y México se configura en función de la producción de la rama automovilística (Aguirre, 2009). Torre y Muñiz (2013) estudiaron la seguridad dentro de los corredores comerciales en Brasil y concluyen que los problemas relacionados con la seguridad son los de mayor impacto y a la vez los de mayor descuido; así mismo definen que uno de los objetivos prioritarios de los corredores es que la zona indicada debe de tener belleza, tranquilidad y orden.

Estudios en corredores comerciales y turísticos establecen comportamientos dispares, en el trabajo de Propin y López (1997), en los cuales se establece la estructura territorial del turismo en el corredor Tijuana-Rosarito-Ensenada, concluyendo con ello que la actividad turística altera el ambiente, genera problemáticas sociales, y se observaron segmentación de diversos espacios de corredor, ya que el desarrollo no fue aprovechado en todo el corredor. López (2002) analizó los flujos turísticos en el corredor de Los Cabos, Baja California Sur, concluyendo que dicho corredor genera intensos flujos de población, turistas, mercancías e información, provocando una disociación de su espacio regional y la pérdida de soberanía nacional.

Muñoz (2000) estudió el corredor turístico Loreto-Nopoló-Puerto Escondido, Baja California Sur, encontrando que la propuesta no fue detonada y el desarrollo que se esperó 
en un principio nunca fue logrado. Gallegos y Segrado (2008) realizaron aproximaciones sobre el corredor turístico Veracruz-Boca del Río, concluyendo que los hechos históricos significativos en el crecimiento del área metropolitana generan un espacio turístico, y su estructura interna presenta los recursos y equipamiento para detonar el corredor previendo buenos resultados.

Una temática de corredores bioceánicos fue estudiada por Inostroza y Bolivar (2004), abordando el problema sobre el debate del tratamiento de los corredores y la necesidad de tomar alternativas y estrategias para el desarrollo de la inserción en los procesos de integración de los países y regiones americanas para el éxito de los corredores.

\section{Planteamiento del problema}

En Ciudad Juárez existen siete corredores comerciales: 1) Ave. Valle del Sol, 2) Ave. Pedro Rosales de León, 3) Ave. Zona Dorada, 4) Ave. Paseo Triunfo de la República, 5) Ave. Tomás Fernández, 6) Ave. Manuel Gómez Morín, y 7) Ave. Juárez-Manzana 14. Cada una cuenta con su director, el cual es el encargado de esgrimir las medidas de colaboración con el Gobierno Municipal y obtener un resultado a los problemas presentados el H. Ayuntamiento de Juárez (2017) y la Secretaría de Turismo (2017). Estos corredores comerciales y turísticos concentran la mayor parte de negocios y servicios de la ciudad, su localización se centra en la zona centro-norte de la ciudad (ver Figura 4), su ubicación responde a la demanda y oferta de espacios comerciales que se han consolidado a través del crecimiento de la ciudad.

Figura 4. Corredores comerciales y turísticos de Ciudad Juárez

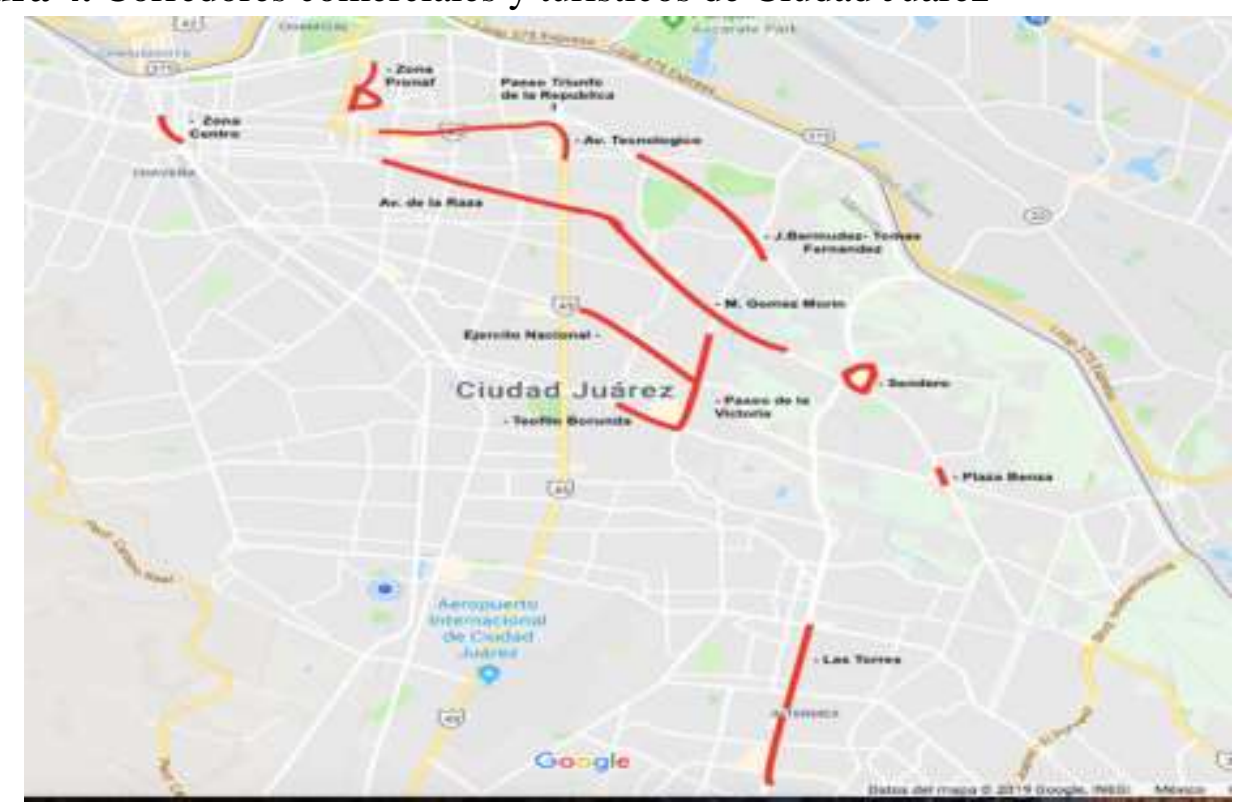

Fuente: Elaboración propia con base en Google Maps (2021). 
Los estudios relacionados con los corredores comerciales son variados y sus aportaciones diversas: Bolaños y Reyes (2020) realizaron un estudio en el corredor comercial Gómez Morín y sus impactos sociales, económicos y ambientales utilizando como base de datos los registros del Sistema 911; otro estudio fue el realizado por Teran y Reyes (2020) donde se analizaron las estrategias tecnológicas para corredores comerciales inteligentes. Ambos estudios permitieron conocer como los corredores comerciales son una estrategia importante para el desarrollo económico y tecnológico de la ciudad.

La llegada de la pandemia del COVID- 19 en los inicios del 2020 provocó un paro comercial y económico en todas las ciudades del mundo, en el caso de Ciudad Juárez fue igual. Además, se le suma el hecho de su ubicación fronteriza con los Estados Unidos, en donde se realizan actividades comerciales y turísticos, que directamente fueron afectados por las restricciones y al cierre indefinido de la frontera. Ante las estrategias de cierres totales a medidas de seguridad, los negocios de los corredores comerciales se vieron en dificultades para mantenerse en el mercado activo, muchos de ellos cerraron y otros cambiaron sus estrategias para llegar a los consumidores y en su mayoría mantuvieron aforos menos de los esperados y disminución drástica de sus ventas. Ante la problemática planteada el objetivo del presente artículo fue el conocer las afectaciones que presentan los corredores comerciales de Ciudad Juárez ante el COVID-19.

\section{ESTRATEGIAS METODOLÓGICAS O MATERIALES Y MÉTODOS}

La presente investigación desarrollada fue de tipo descriptivo y de análisis, debido a que su fin fue conocer las situaciones y actitudes predominantes a través de la descripción exacta de los corredores. De acuerdo a los datos, la investigación fue de naturaleza mixta debido a que implicó el uso de herramientas estadísticas, además del apoyo en los relatos obtenidos en entrevista a profundidad. Este tipo de investigación estudió la relación entre todos los datos obtenidos, tanto cuantitativos como cualitativos, para así conseguir una interpretación precisa de los resultados correspondientes (Hernández, 2018).

La población de estudio es la comunidad de Ciudad Juárez y el objeto de análisis fueron los siete corredores comerciales: la Ave. Valle del Sol, Pedro Rosales de León, Zona Dorada, Ave. Paseo Triunfo de la República, Ave. Tomas Fernández, Ave. Manuel Gómez Morín y Ave. Juárez-Manzana 14 (INEGI, 2018).

Las variables de estudio se definen: cierre de negocio, cambio en la infraestructura, tipos de servicio físico, servicio virtual afectaciones a los empleados. El análisis se realizó en 
el sitio de ubicación de las zonas de ubicación de los corredores comerciales. En cuanto a las afectaciones se utilizó una entrevista a profundidad con el representante de los corredores comerciales en Ciudad Juárez. Las herramientas de recopilación de datos constaron de un instrumento tipo lista de verificación y una entrevista a profundidad.

Para la realización del trabajo de campo se realizó una visita en el sitio, que consistió en un recorrido a los corredores comerciales, visita de revisión física a los negocios ubicados en ambas partes del corredor. Se realizó la entrevista abierta con el C. Lino Andrés Morales Ramírez quien forma parte del comité de Corredores Comerciales de Ciudad Juárez.

La visita física se realizó en los siete corredores de Ciudad Juárez durante los meses de mayo y junio del 2020. Una de las restricciones del estudio fue la existencia de una gran mayoritaria de establecimientos cerrados. Otra fue la negación de empresarios en contestar la encuesta por las medidas de restricción por el COVID-19. Los datos se analizarán utilizando la aplicación de Excel y el software Tableu 15.0.

\section{RESULTADOS Y DISCUSIÓN}

Los resultados se presentan mediante la frecuencia encontrada en la visita a cada corredor comercial para determinar condiciones y establecer las dinámicas y estrategias realizadas durante la pandemia del COVID-19. Los resultados de la entrevista realizada el 16 de mayo del 2020 al director y miembro del Consejo de Corredores Comerciales de Ciudad Juárez, quien explicó las condiciones de cada corredor.

Los resultados reflejan pérdidas en todos los corredores comerciales, el $29 \%$ con mayor afectación es el corredor de la Ave. Juárez, el 21\% fue la Ave. Manuel Gómez Morín y 18\% la Ave. Paseo Triunfo de la República como las de mayor porcentaje (ver Figura 5). Las pérdidas económicas fue el detonante de cierres permanentes de negocios en todas las avenidas. Sin embargo, las estrategias de cambios como fueron: entregas a domicilios, entregas para llevar, ventas por Internet, reducción de foros, adecuaciones a las instalaciones, modificaciones a las reglas de usos, señalizaciones, reglas de acceso y estancia fueron las más frecuentes. Los corredores con mayor número de estrategias relacionadas con el acceso y servicio durante la pandemia fue la Ave. Paseo Triunfo de la República con un 26\%, la Ave Gómez Morín un 20\%, Ave. Tomas Fernández el 16\%, la Ave. Juárez un 13\% como las de mayor porcentaje (ver Figura 6). 
Durante el análisis de los principales factores de los efectos económicos dentro de cada corredor se encontró que la principal afectación en la Avenida Juárez fue debido al cierre de cruce internacional para turistas y visitantes por los puentes internacionales, esto afecto directamente a la avenida ya que es el principal cruce peatonal. El cierre de los puentes evito la llegada de turistas, las visitas de ciudadanos fueron reducida o nulas en días críticos, provocando caída económica de 29\%. Durante el semáforo rojo y la determinación de cierres totales de comercios y negocios no básicos, llevo al cierre masivo en la Ave. Juárez, en donde la principal actividad son artesanías y misceláneos. Los restaurantes redujeron su capacidad y los que estaban listos (tecnológicamente hablando) establecieron los servicios para llevar y entregas a domicilios.

Figura 5. Corredores comerciales que afectan actualmente por el COVID-19

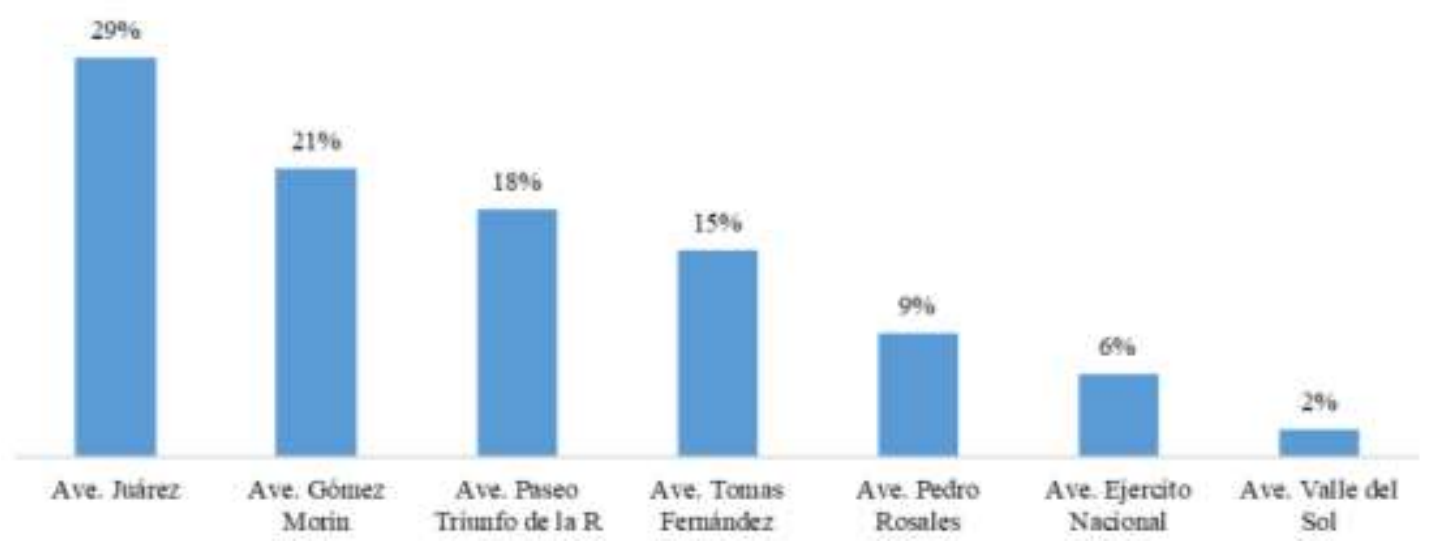

Fuente: Elaboración propia con base en trabajo de campo.

Figura 6. Corredores comerciales con más estrategias ante el COVID-19

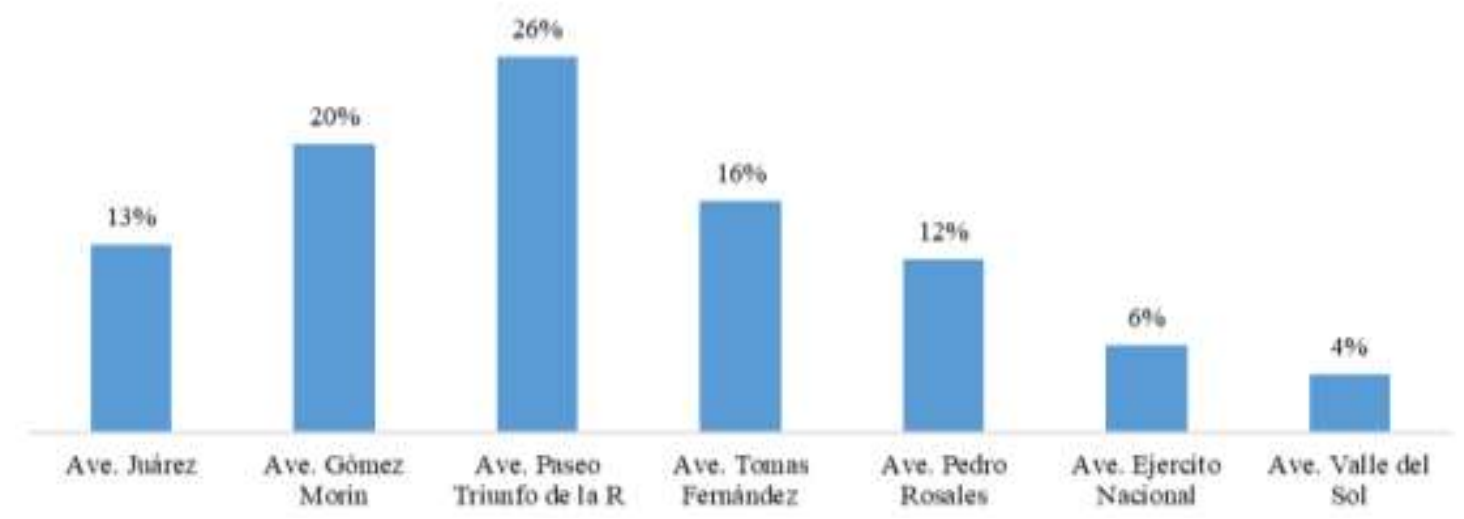

Fuente: Elaboración propia con base en trabajo de campo.

El resto de los corredores tuvieron y mantuvieron servicios reservados y cambios inmediatos para proseguir en funcionamiento, encontrando en todos los corredores 
estrategias en cuanto a la infraestructura, cierres temporales y permanentes, cambios en servicios, uso de aplicaciones virtuales y afectaciones laborales (ver Tabla 3).

Tabla 3. Estrategias comerciales y turísticas de los corredores comerciales de Ciudad Juárez

\begin{tabular}{|c|c|c|}
\hline Factor & Estrategia & $\begin{array}{l}\text { Corredor } \\
\text { Comercial }\end{array}$ \\
\hline Cierre de negocio & Cierre total, cierres parciales & Todos \\
\hline Infraestructura & $\begin{array}{l}\text { Reducción de capacidad aforo, termómetro, } \\
\text { despachador de sanitizante o desinfectante, rutas } \\
\text { de entradas y salidas, separación de estaciones, } \\
\text { construcción de lavabos manos, cuadrillas } \\
\text { desinfectantes constantes, eliminación áreas } \\
\text { especiales, eliminación de eventos, eliminación } \\
\text { áreas de niños }\end{array}$ & Todos \\
\hline Servicio físico & $\begin{array}{l}\text { Entrega a domicilio, lista de espera, reservaciones } \\
\text { horario de estancia (dos horas o menos), cambios } \\
\text { de servicios, servicios a domicilio, servicios para } \\
\text { llevar }\end{array}$ & Todos \\
\hline Servicio virtual & Menús digitales, QR, menús en línea & Todos \\
\hline Empleados & $\begin{array}{l}\text { Despido de empleados (reducción de empleados), } \\
\text { reducción de jornada laboral, nuevas } \\
\text { contrataciones por horas, pérdida de beneficios } \\
\text { laborales, reducción de sueldos, liquidaciones } \\
\text { tempranas }\end{array}$ & Todos \\
\hline
\end{tabular}

Fuente: Elaboración propia con base en trabajo de campo.

Se observa que las estrategias llevadas a cabo en cada corredor hacen referencia a los impactos de inversión, la necesidad de cambiar los tipos de servicios reflejó que no todos los comercios tenían los recursos para hacer cambios en la infraestructura, sin embargo, muchos de ellos hicieron aquellos inmediatos con recursos mínimos. Además, se encontró que en todos los corredores existe afectación económica por el cierre masivo de negocios que no pudieron sostener el pago de impuestos, rentas, suministros, servicios y nómina. La entrevista a profundidad relacionada con las condiciones en las que se encontraba cada corredor comercial permitió observar que, según la ubicación, tipos de servicios y estrategias tomadas, era la situación del corredor:

a) Corredor comercial Ave. Valle del Sol, presentó pérdidas económicas considerables, sin embargo, los negocios que lograron mantenerse abiertos y atendiendo a la clientela encontraron forma de ofrecer sus servicios, aunque a menor escala, su ubicación de 
cruce a zonas habitacionales le permitió continuar con ventas menores pero constantes (ver Tabla 4).

Tabla 4. Corredor comercial, Avenida Valle del Sol

\begin{tabular}{|l|}
\hline Avenida Valle del Sol \\
El motivo de este auge comercial en la zona de Valle del Sol se debe al incremento poblacional \\
que cuentan con un total de 33 locales comerciales. Al comienzo de 2020 se abrieron al menos \\
ocho nuevos locales en Plaza La Cantera. La primera plaza en construcción se ubica enfrente \\
de la tienda de conveniencia Oxxo, en la cual se construirán 13 locales comerciales. Se \\
encuentra la segunda plaza que albergará 20 locales y se ubica a un costado de una tienda \\
Superette. El presidente de la AMPI Pablo Hernández Sáenz añadió que por la cantidad de \\
gente que vive en la zona y que es de clase media, este punto comercial es sustentable para que \\
los inversionistas tengan la confianza de apostarle a esa avenida. Valle del Sol es una de las \\
zonas donde ya no se permiten más permisos para nuevos comercios, sin embargo, los juarenses \\
continúan viendo esa área atractiva para abrir un negocio \\
\hline Afectaciones por el COVID-19 \\
\hline Para los resultados del corredor Valle del Sol, se requirió tener que ir a la zona para observar \\
el comportamiento de las plazas y locales, encontrando que los restaurantes y puestos de \\
comida entregan la comida por autoservicio y a domicilio bajando sus ganancias un $2.2 \%$. La \\
Avenida Valle del Sol se caracteriza por ser zona de restaurantes, ventas de comercio y puestos \\
de comida pequeña, por lo que las ventas continúan, tomando medidas sanitarias como \\
garantizar la rotulación del personal, usando guantes, cubre bocas, gel antibacterial, \\
desinfectando las superficies que se tocan con frecuencia. Continúa comprando gente, continúa \\
habiendo poco de ganancia en las ventas.
\end{tabular}

Fuente: Elaboración propia con base en trabajo de campo.

b) Corredor comercial Ave. Pedro Rosales de León. El corredor durante el 2019 fue remodelado y cambio su infraestructura. Durante la pandemia presentó condiciones económicas serios ya que restaurantes y plazas fueron cerradas en su totalidad. En este sentido, se tuvo un efecto del $9.1 \%$ de bajo rendimiento. (ver Tabla 5).

Tabla 5. Corredor comercial, Avenida Pedro Rosales de León

\section{Avenida Pedro Rosales de León}

El motivo de este auge en la Ave. Pedro Rosales de León con el crecimiento de Nuevas plazas comerciales, con diseños arquitectónicos modernistas, están cambiando el paisaje de algunas avenidas de la ciudad. Con características distintas al conjunto comercial tradicional juarense, la avenida ahora combina oficinas, negocios y hasta espacios de convivencia.

\section{Afectaciones por el COVID-19}

Cierre total de plazas comerciales, pérdidas económicas en las ventas, despidos de empleados en todos los negocios.

Se utilizan medidas básicas como cubre bocas, afectando la variable económica debido que se observa muy sola la zona.

Fuente: Elaboración propia con base en trabajo de campo. 
c) Corredor comercial Zona Dorada. La actividad comercial continuó, los negocios no cerraron, se siguió con los mismos servicios y ventas durante la pandemia. Se observó un flujo abundante de tráfico por la zona, un aumento en el crecimiento económico en tiendas mayoristas, debido a las fechas importantes que los caracteriza (ver Tabla 6).

Tabla 6. Corredor comercial, Zona Dorada

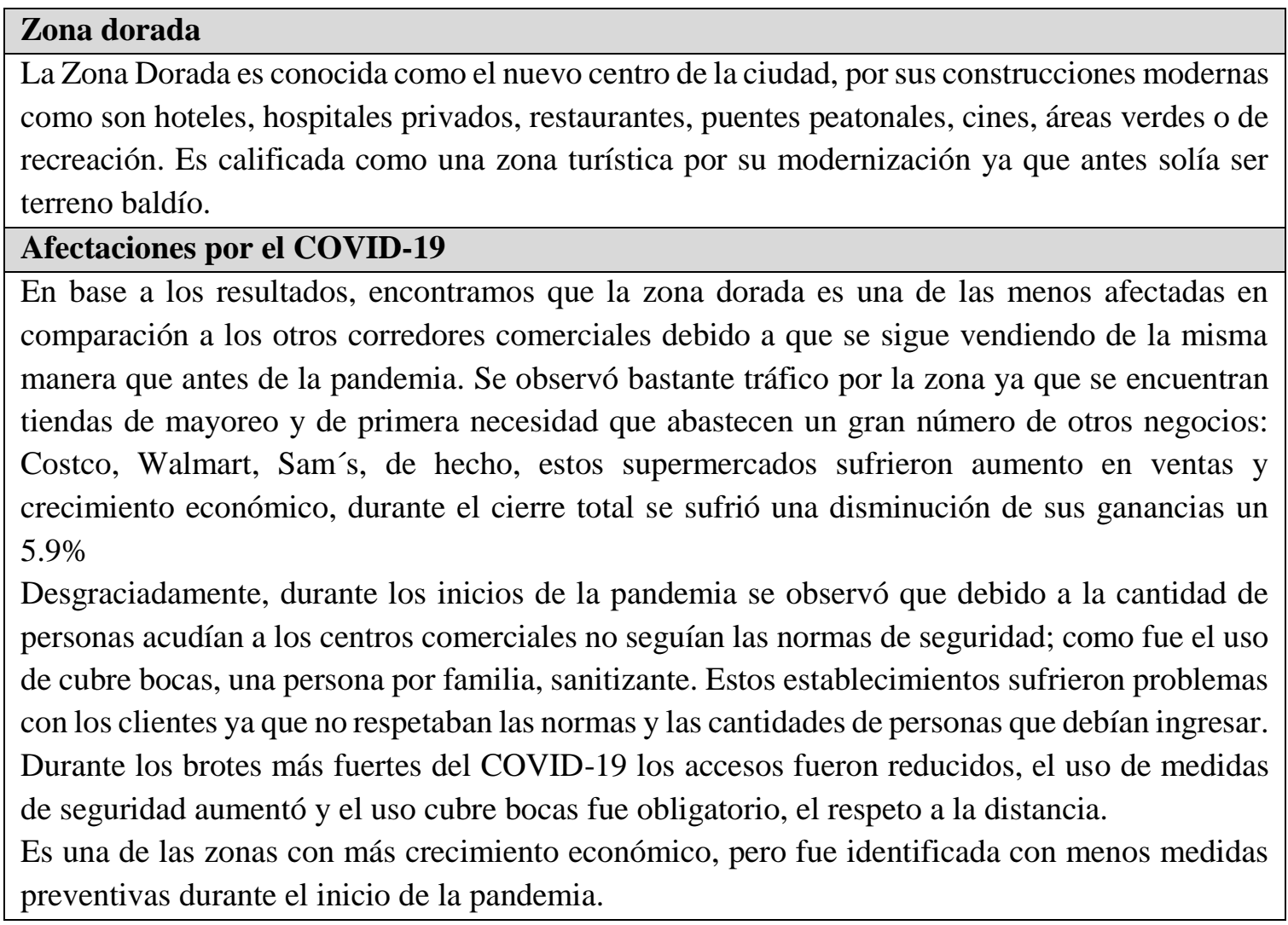

Fuente: Elaboración propia con base en trabajo de campo.

d) Corredor comercial Ave. Paseo Triunfo de la República. En cuestión económica se observa que el comercio descendió un $17.9 \%$ en comparación a otros corredores. En lo que más se afectó al corredor fue la zona hotelera, debido a que solo permiten un porcentaje mínimo de huéspedes, dejando al personal con sueldos bajos e incluso con bastantes liquidaciones (ver Tabla 7).

Tabla 7. Corredor comercial. Avenida Paseo Triunfo de la República

\section{Avenida Paseo Triunfo De La República.}

La Ave. Paseo Triunfo de la Republica es la zona que se caracteriza por ser zona hotelera. Agencia de viajes, bancos, ópticas y centros comerciales. Actualmente es conocida por la cantidad de comercio que vende la zona.

El representante del corredor comercial reconoce el esfuerzo y la gran dedicación que ponen todos los elementos de la Policía Municipal en sus labores, destacando su actitud, disponibilidad y atención que brindan en cada recorrido en los diversos comercios, ante el COVID-19. 


\section{Afectaciones por el COVID-19}

El corredor comercial es una de las zonas con más vigilancia y seguridad ante el COVID19, por lo que queda prohibido no usar las protecciones ante el virus. En cuestión económica se observa que el comercio descendió, pero no en comparación a otros corredores comerciales disminuyendo al $17.9 \%$. La ubicación del corredor y la construcción de las paradas de la nueva línea 1 BRT causaron problemas de tráfico, acceso a los negocios y las perdidas han sido cuantiosas.

Lo que más afecto al corredor, es la zona hotelera, debido a que solo permiten un porcentaje mínimo de huespedes, dejando al personal con sueldos bajos e incluso con bastantes liquidaciones.

Fuente: Elaboración propia con base en trabajo de campo.

e) Corredor comercial Ave. Tomas Fernández. El corredor comercial es reconocido por la cantidad de restaurantes y bares, al ser no necesarios se tuvo una pérdida cuantiosa, durante el 2020 se encontraban muchos negocios cerrados, los que se mantuvieron abiertos presentaron perdidas en las ventas, muchos realizaron cambios en el tipo de servicios al utilizar los servicios de Uber Eats o entrega rápida, los centros sociales permanecieron cerrados, así como escuelas y establecimientos (ver Tabla 8).

Tabla 8. Corredor comercial, Avenida Tomas Fernández

\begin{tabular}{|l|}
\hline Avenida Tomas Fernández \\
\hline $\begin{array}{l}\text { La avenida Tomas Fernández es una de las zonas caracterizada de mayo prestigio, dando lugar } \\
\text { a la zona maquilera, a antros, centros sociales, restaurantes. }\end{array}$ \\
\hline Afectaciones por el COVID-19 \\
\hline Basándonos en la afectación de la Ave. Tomas Fernández se disminuyó un $15.3 \%$ la actividad \\
económica debido a que el personal de las maquilas ha rotado a sus empleados, \\
disminuyéndoles el sueldo al 50\%. En los restaurantes y bares, la producción bajo, basándose \\
a que solo se encuentra abierto por Uber Eats o entrega rápida, los aforos durante los meses \\
más altos de la pandemia fueron de cero, los centros sociales permanecieron cerrados en su \\
totalidad, así como escuelas y establecimientos.
\end{tabular}

Fuente: Elaboración propia con base en trabajo de campo.

f) Corredor comercial Ave. Juárez. La avenida Juárez es el corredor que más ha sufrido económicamente debido a su ubicación en el centro histórico de la ciudad y al ser la avenida para el cruce hacia los Estados Unidos de Norte América, el cierre de los negocios y la falta de visitantes fue disminuido casi a cero y afecto a toda la actividad comercial. La cantidad de centros cambiarios, bares, restaurantes, artesanías y misceláneos fueron cerrados en su totalidad por lo que su economía disminuyó al $29.20 \%$ (ver Tabla 9). 
Tabla 9. Corredor comercial, Avenida Juárez

\begin{tabular}{|l|}
\hline Avenida Juárez \\
\hline La Ave. Juárez se ha convertido en una de las zonas de la localidad que más recursos ha recibido \\
de fondos municipales, estatales y federales para remodelaciones y ampliación de banquetas, \\
los cuales en el último mes ha caído a causa del coronavirus. Ya no se permite nuevos \\
comercios y en cuanto al negocio informal está prohibido, porque no hay dónde se pongan, no \\
existen zonas. La avenida Juárez es otra zona donde está restringido el permiso, no hay \\
permisos nuevos, sólo se renuevan los que ya están y tienen arriba de 15 años. \\
\hline Afectaciones ante el COVID 19 \\
\hline $\begin{array}{l}\text { La avenida Juárez es el corredor que más ha sufrido económicamente, afectando fuertemente } \\
\text { por ser frontera, el público extranjero, disminuyó el trabajo en centros cambiarios, los bares } \\
\text { que se localizan ahí están cerrados, por lo que su economía disminuyó al } 29.20 \% \text {, las farmacias } \\
\text { y ciertas clínicas fueron las únicas abiertas durante la pandemia. }\end{array}$ \\
\hline
\end{tabular}

Fuente: Elaboración propia con base en trabajo de campo.

g) Corredor comercial Ave. Gómez Morín. Actualmente es uno de los corredores de mayor actividad económica, la inversión en restaurantes, bares, centros comerciales y servicios, la mantienen como una de las más importantes. Durante la pandemia fue una de las afectadas y se tuvieron cierres de muchos negocios. Adicional se construye la ruta del BRT de transporte público que ocasionó problemas de tránsito y acceso a negocios. Los restaurantes que se mantuvieron cambiaron sus servicios a entregas a domicilio y servicios de Uber Eats y entregas rápidas. Además, se realizaron rebajas para que el cliente continuara comprando (ver Tabla 10).

Tabla 10. Corredor comercial, Avenida Gómez Morín

Avenida Gómez Morín

La Ave. Gómez Morín es el corredor comercial más importante, caracterizado por el alto comercio y ventas que se encuentra en toda la zona. Lo que más sobresale, son los antros y bares localizados en este corredor, donde cada fin de semana se llena de jóvenes que pasan horas de diversión.

Restaurantes de lujo, así como puestos de comida chatarra inundan las calles de la Ave. Gómez Morín fortaleciendo el crecimiento de la economía.

\section{Afectaciones ante el covid-19}

Existe mucha vigilancia en todo el corredor por los tipos de negocios que se localizan, la llegada de jóvenes es inevitable durante los fines de semana y durante la semana los restaurantes son visitados con mucha frecuencia. Durante la pandemia muchos mantuvieron sus servicios aun existiendo la recomendación de quedarse en casa. Por su importancia económica es el corredor con el segundo lugar en afectación ante el COVID-19, debido al alto crecimiento de pérdidas en las ventas de comida, obteniendo un $20.60 \%$ de afectación.

Actualmente los restaurantes que siguen laborando, son en base al servicio a domicilio o por transporte rápido, el aforo bajo a lo permitido por la Ley y se generaron descuentos en los consumos para continuar con las ventas para que el cliente compre.

Fuente: Elaboración propia con base en trabajo de campo. 
Los hallazgos revelan las acciones tomadas ante la pandemia del COVID-19, los corredores comerciales dieron respuestas diversas al riesgo asociado hacia la idea de la incertidumbre en la que todos se encontraban. Además, dieron respuestas desde la visión de la vulnerabilidad y la capacidad de ajustarse a las vicisitudes mediante estrategias que les permitieron mantenerse durante el 2020 y prosiguen durante el 2021.

La capacidad resiliente de los empresarios para mantenerse con vida económica y adaptarse a los cambios mediante la diversificación en los servicios da evidencia de que la adaptación en los momentos de crisis es vital para mantenerte en el mercado (Arciniega, 2005). Con la adecuación a las condiciones de la pandemia se logra resistir a la destrucción, es decir, preservar la integridad en circunstancias difíciles; la actitud de reaccionar positivamente a pesar de las dificultades (Vaniestendael, 1994, citado por Fiorentino, 2008).

Aun y en las condiciones más adversas los corredores comerciales se han logrado mantener, si bien no todos los negocios subsistieron a los cierres masivos, muchos de ellos crearon estrategias que les permitieron continuar en las condiciones más adversas y con pocas posibilidades de éxito. Sin embargo, son evidencia de que, si se quiere, solo es pertinente adaptarse de inmediato y no darse por vencidos.

Los resultados permiten observar que el corredor más afectado es el del centro histórico Ave. Juárez, sin embargo, una de las afectaciones mayores es por la dependencia de turistas y visitantes, lo que llevo al cierre masivo de negocios, en contrario a los ubicados en el crecimiento urbano.

\section{CONCLUSIÓN O CONSIDERACIONES FINALES}

Se concluye que el COVID -19 fue y es, un factor alarmante que continúa afectando a todos los sectores económicos del mundo. Los corredores comerciales como parte estratégica de las ciudades continúan albergando el conjunto de negocios y comercios para el beneficio de la comunidad. Indiscutiblemente, muchos de estos corredores perdieron elementos claves que no les permitieron sobrevivir, imponiéndoles cierres y/o en el mejor de los casos sobrevivir en lo mínimo. La existencia de diversas restricciones para mantenerse en el mercado, la falta de apoyos económicos, reducción de rentas o ayudas fiscales, para los comerciantes los llevo también al cierre masivo de negocios. Las estrategias implementadas se han convertido entonces en paliativos al dolor de no poder 
continuar con servicios "normales"; sin embargo, son una alternativa que les da la oportunidad de continuar.

Es de admirar como los micros, pequeños, medianos y grandes empresarios aceptaron las condiciones de prevención de contagios; los medios implementados fueron erogados de su propia inversión económica para continuar y buscan reducir o eliminar brotes, sin afectar la economía del sector. Aunque las estrategias no fueron acciones complejas en su mayoría, la toma de temperatura, tapetes satirizantes, lava-manos, disminución del aforo, cortinas de vidrio, plástico y continuar con los pedidos de entrega a domicilio mediante llamadas telefónicas y de las aplicaciones tecnológicas, evitaron y previnieron en su mayoría los contagios, dando con ello una alternativa de continuar con la actividad económica de la ciudad.

Los corredores comerciales y turísticos en Ciudad Juárez dan evidencia entonces de que las acciones conjuntas logran más que aquellas en lo individual; lo pequeño y constante permite abonar a sobrevivir con los efectos masivos de un virus letal como lo es el COVID-19.

\section{LISTA DE REFERENCIAS}

Aguirre, S. M. (2009). La rama automovilística y los corredores comerciales del TLCAN. Comercio exterior, 59(5), 370-378. Recuperado de: http://revistas.bancomext.gob.mx/rce/magazines/125/3/370_Serafin_Maldonado. pdf.

Ander-Egg, E. (2000). Metodología y Práctica de la Animación Sociocultural. Editorial CCS. Recuperado de: https://www.todostuslibros.com/libros/metodologia-ypractica-de-la-animacion-sociocultural_978-84-8316-365-8.

Arciniega, J. de D. (2005). La resiliencia. Una nueva perspectiva en psicopatología del desarrollo. Revista de Psicodidáctica, 10(2), 61-80. Recuperado de: https://ojs.ehu.eus/index.php/psicodidactica/article/view/190.

Bolaños-Ramírez, M.A., \& Reyes-Escalante, A.-Y. (2020). Corredor comercial gómez morín y sus impactos sociales, económicos y ambientales: Atlas de riesgo y georreferencial del sistema 911. Revista TURISMO: Estudos e Práticas, 9(Temático 1), 1-9. Recuperado de: http://natal.uern.br/periodicos/index.php/RTEP/article/view/1988. 
Calaméo (2021). Glosario De Términos Turísticos. Recuperado de: https://es.calameo.com/books/006166513a707d91aee89.

Castro L., R. (2020). Coronavirus, una historia en desarrollo. Revista médica de Chile, 148(2), 143-144. DOI: https://doi.org/10.4067/s0034-98872020000200143

Collison, J. (2020). The Impact of Online Food Delivery Services on Restaurant Sales. $\mathrm{PhD}$ Dissertation, Stanford University. Recuperado de: https://web.stanford.edu/ leinav/teaching/Collison.pdf.

Dirección General de Epidemiología (2021). COVID-19, México: Datos epidemiológicos. Recuperado de: https://covid19.sinave.gob.mx/.

Fiorentino, M. T. (2008). La construcción de la resiliencia en el mejoramiento de la calidad de vida y la salud. Suma Psicológica, 15(1), 95-114.

Gallegos, O., y Segrado, R. G. (2008). Organización espacial del corredor turístico Veracruz-Boca del Río. Teoría y Praxis, 5, 171-186. Recuperado de: http://risisbi.uqroo.mx/handle/20.500.12249/629.

Google Maps (2021). Mapa de Ciudad Juárez. Recuperado de: https://www.google.com.mx/maps/@31.7593738,-106.4505817,15z.

Hernández, R. H. (2018). Metodología de la investigación: Las rutas cuantitativa, cualitativa y mixta. McGraw Hill.

INEGI (2021a). COVID-19 Tablero México. COVID - 19 Tablero México. Recuperado de: https://datos.covid-19.conacyt.mx/index.php.

INEGI (2021b). MexiCOVID: Covid-19 en México. Recuperado de: https://mexicovid19.app/.

Inostroza, L., y Bolivar, A. (2004). Corredores bioceánicos: Territorios, políticas y estrategias de integración subregional. Análisis Económico, XIX(41), 153-174. Recuperado de: https://www.redalyc.org/pdf/413/41304107.pdf

Kuckertz, A., Brändle, L., Gaudig, A., Hinderer, S., Morales Reyes, C. A., Prochotta, A., Steinbrink, K. M., \& Berger, E. S. (2020). Startups in times of crisis - A rapid response to the COVID-19 pandemic. Journal of Business Venturing Insights, 13, e00169. DOI: https://doi.org/10.1016/j.jbvi.2020.e00169.

López, Á. L. (2002). Análisis de los flujos turísticos en el corredor Los Cabos, Baja California Sur. Investigaciones Geográficas. Bolefin del Institufo de Geografia, 47, 131-149. Recuperado de: http://www.scielo.org.mx/pdf/igeo/n47/n47a9.pdf 
Moreno, T. (2015). Plan maestro del corredor logístico industrial automotriz del Bajío. Quivera, 17(1), 13-34. Recuperado de: https://www.redalyc.org/pdf/401/40140031002.pdf

Muñoz, R. A. (2000). El corredor turístico Loreto-Nopoló-Puerto Escondido, Baja California Sur, en el contexto de los centros integralmente planeados. Cuadernos de Turismo, 5, 53-68. Recuperado de: https://revistas.um.es/turismo/article/view/22741

Organización de los Estados Americanos (OEA) (2021). Desarrollo de Corredores de Comercio y Reducción de la Vulnerabilidad del Sector Transporte. Recuperado de: https://www.oas.org/nhp/transvial.html

Organización Mundial del Comercio (OMC) (2021). La OMC informa a los Miembros y observadores sobre iniciativas y análisis relacionados con la COVID-19. Recuperada de https://www.wto.org/spanish/news_s/news21_s/covid_12oct21_s.htm

Organización Mundial del Turismo (OMT) (2021). World Trade Organization-Global trade. Recuperado de: https://www.wto.org/index.htm.

Organización Mundial de la Salud (OMS) (2021). Brote de enfermedad por el Coronavirus (COVID-19)-OPS/OMS. Recuperado de: https://www.paho.org/es/temas/coronavirus/brote-enfermedad-por-coronaviruscovid-19.

Propin, E., Sánchez-Crispín, A., \& López, A. (1997). Situación geográfico-económica del turismo en el extremo norte de América Latina: El corredor Tijuana-RosaritoEnsenada. Revista de Geografía Norte Grande, 24, 257-261. Recuperado de: https://www.researchgate.net/profile/Enrique-

Propin/publication/266883002_Situacion_geograficoeconomica_del_turismo_en_el_extremo_norte_de_America_Latina_El_corredor _Tijuana-Rosarito-Ensenada/links/55539a2e08ae6fd2d81f20dc/Situaciongeografico-economica-del-turismo-en-el-extremo-norte-de-America-Latina-Elcorredor-Tijuana-Rosarito-Ensenada.pdf

Romero, Fernando (2011). Propuesta del Corredor Cultural Chapultepec en la Ciudad de México. Recuperado de https://www.archdaily.mx/mx/770422/mexico- 
parque-elevado-chapultepec-proyecto-catalizador-para-generar-comunidad-en-

$\underline{\text { la-ciudad-de-mexico?ad medium=widget\&ad name=recommendation }}$

Secretaría de Gobernación (2019). Plan Nacional de Desarrollo. Recuperado de: https://www.dof.gob.mx/nota_detalle.php?codigo=5565599\&fecha=12/07/2019. Secretaría de Turismo (2017). Se desarrolla el Programa Corredores Turísticos Artesanales en 11 estados de la República. Recuperado de: http://www.gob.mx/bienestar/prensa/se-desarrolla-el-programa-corredoresturisticos-artesanales-en-11-estados-de-la-republica.

SER (2021). La pérdida de libertad con las restricciones. Recuperado de: https://cadenaser.com/emisora/2021/02/11/radio_penafiel/1613044974_956504. $\underline{\mathrm{html}}$.

Torre, J. Á., y Muñiz, D. R. (2013). Riesgo y percepción en el desarrollo de la imagen turística de Brasil ante los mega-eventos deportivos. PASOS. Revista de Turismo y Patrimonio Cultural, 11(3), 147-154. DOI: https://doi.org/10.25145/j.pasos.2013.11.046. 